\title{
Binary Paths to Type Ia Supernovae Explosions: the Highlights
}

\author{
Lilia Ferrario \\ Department of Mathematics \\ Mathematical Sciences Institute \\ The Australian National University, ACT 0200 \\ Australia \\ email: Lilia.Ferrario@anu.edu.au
}

\begin{abstract}
This symposium was focused on the hunt for the progenitors of Type Ia supernovae (SNe Ia). Is there a main channel for the production of SNe Ia? If so, are these elusive progenitors single degenerate or double degenerate systems? Although most participants seemed to favor the single degenerate channel, there was no general agreement on the type of binary system at play. An observational puzzle that was highlighted was the apparent paucity of supersoft sources in our Galaxy and also in external galaxies. The single degenerate channel (and as it was pointed out, quite possibly also the double degenerate channel) requires the binary system to pass through a phase of steady nuclear burning. However, the observed number of supersoft sources falls short by a factor of up to 100 in explaining the estimated birth rates of SNe Ia. Thus, are these supersoft sources somehow hidden away and radiating at different wavelengths, or are we missing some important pieces of this puzzle that may lead to the elimination of a certain class of progenitor? Another unanswered question concerns the dependence of SNe Ia luminosities on the age of their host galaxy. Several hypotheses were put forward, but none was singled out as the most likely explanation.

It is fair to say that at the end of the symposium the definitive answer to the vexed progenitor question remained well and truly wide open.
\end{abstract}

Keywords. binaries: close - stars: cataclysmic variables, supernovae, white dwarfs - cosmology: observations

\section{Introduction}

The long standing problem of establishing which objects produce SNe Ia is often referred to as the "progenitor problem", and the main aim of this symposium was to shed some light on this decades old question.

It is widely acknowledged that SNe Ia are generated by thermonuclear disruptions of carbon-oxygen (CO) white dwarfs (WDs). Thus, according to the most commonly accepted view, a WD must either accrete enough matter from its companion or it must merge with its companion. Hence the following two principal channels for the formation of SNe Ia.

(a) Single degenerate (SD) channel: a WD accretes mass from a non-degenerate companion star, reaches the Chandrasekhar limiting mass, and explodes. This channel was first proposed by Whelan \& Iben (1973). Alternatively, a WD accumulates a large enough mass of helium on top of its $\mathrm{CO}$ core either via accretion or through the burning of $\mathrm{H}$ into He. The helium layer ignites and creates a burning front propagating outwards through the helium shell while an inward compressional wave ignites the CO causing a detonation which propagates outwards ("double-detonation" model, Woosley \& Weaver 1994). 
(b) Double degenerate (DD) channel: two WDs merge to form a single WD, achieving Chandrasekhar's limiting mass, which then explodes. This pathway was first proposed by Iben \& Tutukov (1987) and Webbink (1984).

The SD channel received a major boost following the discovery of supersoft X-ray sources (SSSs, e.g. Trümper et al. 1991), which are binary systems consisting of a WD which accretes hydrogen at a rate that is high enough $\left(\geqslant 10^{-7} \mathrm{M}_{\odot} \mathrm{yr}^{-1}\right)$ to allow steady nuclear burning over a period of $\sim 10^{5}-10^{6}$ years. Thus, if these WDs can accumulate $\sim 0.1-0.4 \mathrm{M}_{\odot}$ of material during their nuclear burning phase, they could end up as SNe Ia.

In the case of stable Roche lobe overflow (RLOF) the donor does not need to be a hydrogen-rich main sequence (MS) or giant-like star. It could be a non-degenerate or a semi-degenerate helium-burning star, or even a helium WD (e.g. Iben et al. 1987).

Another interesting avenue was presented by Soker (this volume). This consists of a merger between a WD with the hot core of a massive AGB star during common envelope (CE) evolution.

Needless to say, the symposium was entirely focused on discussions on the pros and cons of the two main channels (DD versus SD) both from observational and theoretical points of view.

\section{Single Degenerate Channel}

In the SD channel, a massive WD needs to steadily burn hydrogen and increase its mass to reach the Chandrasekhar limit.

Symbiotic stars (SSs - see the review by Mikolajewska, this volume) are long orbital period $\left(\sim 10^{2}-10^{3}\right.$ days) interacting binaries in which a hot WD orbits around a cool $\mathrm{M}$ giant or a Mira variable. They are usually immersed in an emission nebula and there is no direct evidence for the existence of an accretion disk.

Mass transfer occurs via Bondi-Hoyle-Littleton (BHL) wind accretion, although the recent detection of ellipsoidal variability in some SSs with $P_{\text {orb }}<10^{3}$ days suggests that some of the giant donors are close to filling their Roche lobes. During quiescence, SSs undergo stable hydrogen burning on the WD. Thermonuclear nova explosion are rare in classical SSs (9 objects out of $\sim 200)$.

Recurrent novae (RNe, see the review by Anupama, this volume) exhibit nova explosions with mass ejection of $10^{-7}-10^{-6} \mathrm{M}_{\odot} \mathrm{yr}^{-1}$ on recurrence timescales of $\sim 10-10^{2}$ years . RNe can be divided into two subclasses: (i) systems with a dwarf secondary and (ii) systems with a red giant secondary. Those with a giant secondaries, such as RS Oph, T CrB, V3890 Sgr and V745 Sco, bear many similarities with the symbiotic stars. Thus, these are often referred to as symbiotic recurrent novae (SyRNe). Recent observations of ellipsoidal variations in all SyRNe, except for RS Oph, indicate that mass accretion in most SyRNe occurs via RLOF.

Interestingly, the theoretical calculations of Mohamed \& Podsiadlowski (this volume) show that standard wind accretion is not adequate to explain the observations of SSs. Thus, they propose a model where the wind acceleration region of the evolved star occurs close to its Roche lobe. Thus, the wind material can fill the Roche lobe and matter can be transferred to the hot star through the inner Lagrangian point. The mass transfer rate is estimated to be up to 100 times larger than that expected from BHL wind accretion.

The orbital parameters are known for only two SyRNe: T CrB and RS Oph (e.g. Nelson et al. 2011, Belczynski \& Mikolajewska 1998). The orbital periods are amongst the shortest observed for SSs: 227 and 453 days, respectively. The systems T $\mathrm{CrB}$ and RS Oph are also the only SSs with the hot and massive WD $\left(1.1-1.4 \mathrm{M}_{\odot}\right)$ being more 
massive than the cool giant companion whose mass is only $\sim 0.6 \mathrm{M}_{\odot}$ (much lower than that of any other symbiotic giant). Mukai et al. (this volume) and Orio, Nelson \& Rauch (this volume), show how X-ray observations of accreting WDs can be used to set lower limits to the mass, temperature, and chemical composition of WDs in RNe.

Thus, observations indicate that the masses of the WDs in a fraction of SyRNe are sufficiently high to allow them to become SNe Ia if enough material is accreted (see Kato, this volume). The low mass of the nova ejecta and the absence of WD processed material indicate that the WDs in these systems burn steadily the accreted material and during eruption they do not expel more matter than they have accreted. Hence, the mass of the WD can grow and SyRNe could in principle provide a good avenue for the production of SNe Ia (see also the calculations of Starrfield et al., this volume).

However, it is not at all clear whether these WDs were either (i) relatively low-mass CO WDs that have accreted a significant amount of mass during binary evolution or (ii) they were massive ONeMg WDs at birth. In the first (i) case, an SN Ia event becomes possible. In the second (ii) case, the ONeMg WD will undergo an accretion induced collapse (AIC) and become a neutron star (Yoon, Podsiadlowski \& Rosswog 2007). In fact, population synthesis calculations have shown that this route can provide a much better match to the period distributions of long period binary millisecond radio pulsars (MSPs) than those that result from core-collapse SNe II and subsequently go through a low-mass X-ray binary phase (Hurley et al. 2010).

Regardless of the final outcome, massive WDs accreting and burning hydrogen at high rates [i.e. steady nuclear burning WDs, (NBWDs)] are hot, bright $\left(L \sim 10^{38} \mathrm{erg} \mathrm{s}^{-1}\right)$, and should be observable as SSSs. However, only $\sim 380$ SSSs have been discovered in external galaxies which is a number that is up to two orders of magnitudes too small to match the observed SNe Ia rate (Di Stefano, this volume). Thus, if the observed population of SSSs is so much smaller than required, we are led to hypothesize that either (i) the $\mathrm{SD}$ channel is just a very minor route to the production of SNe Ia; or (ii) most of the time these NBWDs do not look like SSSs (Di Stefano, this volume). In the context of (ii), Nielsen, Dominik \& Nelemans (this volume) have built a model where obscuration by gas within the system hides most of the nuclear burning phase of these WDs making them practically unobservable to current X-ray telescopes. But then we should try to answer the following question: "If the energy generated by steady nuclear burning does not emerge at X-ray wavelengths, in which wave-bands should these sources be looked for?"

Wang \& Han (this volume) used Eggleton's stellar evolution code with the optically thick wind assumption to study the SD channel where the donor is a helium star (either main-sequence or a He sub-giant). They found that this channel can produce the class of SNe Ia with short delay times $(<100 \mathrm{Myr})$. They also predict that the surviving companion would be characterized by a spatial velocity of $\geqslant 400 \mathrm{~km} / \mathrm{s}$ (

\subsection{Super-Chandrasekhar SNe Ia}

Recent observations of a class of super-luminous SNe Ia indicate that some exploding WDs may have masses of up to $2.4-2.8 \mathrm{M}_{\odot}$, well above the Chandrasekhar mass limit (e.g. Howell et al. 2006). One of these objects is SN Ia 2009dc whose late-phase observations were reported by Yamanaka et al. (this volume). Hachisu et al. (this volume) presented a binary scenario where accreting WDs can reach masses as high as $2.4 \mathrm{M}_{\odot}$ supported by rotation against collapse and explosion. Magnetic-dipole radiation would lead to spin-down on time-scales of $<10^{9}$ years in WDs with $M \geqslant 1.6 \mathrm{M}_{\odot}$ and $>10^{9}$ years in WDs with $1.38<M / M_{\odot}<1.5$. The range in spin-down time-scales would thus produce both the prompt and the delayed variety of SNe Ia (see $\S 5$ ). 
This scenario is similar to that recently reported by Di Stefano, Voss, \& Claeys (2011). Their model predicts that by the time an SN Ia explosion occurs, the donor star could have had sufficient time to lose its envelope and perhaps become a WD. This would have the benefit of reducing the impact that a hydrogen-rich donor would have on the SN characteristic signatures. Before the explosion these systems could appear either as super-Chandrasekhar mass WDs with a WD companion or as CVs. After the explosion, the model forecasts the existence of a population of either WDs, or low-mass stars with high spatial velocities. This scenario would also have the added benefit of explaining the existence of single low-mass WDs $\left(\mathrm{M}<0.5 \mathrm{M}_{\odot}\right)$ as the remnants of giant-branch donors whose envelopes were stripped away by the supernova explosion (Justham et al. 2009).

\subsection{Runaway Donor Stars in the SD Channel}

The SD route to SNe Ia predicts that the donor star should survive an SN Ia explosion (e.g. Marietta, Burrows \& Fryxell 2000). Consequently, if such a star could be found, this would provide substantial evidence in support of this channel.

Kerzendorf et al. (this volume) have argued that a left over donor star could be identified by its space velocity. Furthermore, if one assume RLOF, the donor star is expected to be tidally locked to the rotation period of the system at the time of explosion and thus be rapidly rotating.

They analyzed 79 stars near the center of the remnant of SN 1006 (at the distance of $2.2 \mathrm{kpc}$ ), but found no stars, down to half a solar luminosity, displaying an unusual space or significant rotational velocities (down to $<20 \mathrm{~km} \mathrm{~s}^{-1}$ ). They also re-examined a sub-giant star (Star G) which was identified by Ruiz-Lapuente et al. (2004) as the donor star of Tycho's 1572 SN Ia. Kerzendorf et al. argued that although Star G exhibits unusual kinematics, it is off-center and shows no rotation. Furthermore, they could not confirm the presence of nickel in its atmosphere as reported by Hernández et al. (2009). Thus, they argue that Star G is not likely to be associated with the SN event.

Ruiz-Lapuente et al. (this volume) also conducted a survey down to $R=15$ of the stars within a 4 arcmin radius around the remnant of SN 1006 for a possible surviving binary donor. The limiting magnitude of the survey would have allowed the detection of red-giant type stars, but their results were also inconclusive.

Podsiadlowski (2003) reports that to exclude a sub-giant companion, it is necessary to consider much fainter objects than previously forecast. For example, one should look for stars with a luminosity of $0.1 \mathrm{~L}_{\odot}$ in a 1,000 year old remnant, or $0.01 \mathrm{~L}_{\odot}$ in a 10,000 year old remnant. Thus, deeper surveys of SN Ia remnants may be needed to look for these runaway donor stars.

However, if these deeper surveys still cannot identify a suitable candidate, this would indicate that other progenitor scenarios, such as the DD channel (see §3), are at work or that there is a long time delay between the accretion phase and the SN Ia explosion that would allow the donor to become a He WD, as expected in some models (e.g. Di Stefano, Voss, \& Claeys 2011).

\subsection{Classical CVs and WD Masses: A Case for Failed SNe Ia?}

Theoretical simulations and observations indicate the WD mass in CVs decreases over time due to nova eruptions. However, Zorotovic et al. (2011) have discovered that recent high-precision measurements of WD masses in a large number of CVs point to primary masses which are significantly higher $\left(0.8-0.9 \mathrm{M}_{\odot}\right)$ than the average mass of isolated WDs or of WDs in pre-CVs $\left(\sim 0.6 \mathrm{M}_{\odot}\right)$. Taken at face value, these observations may indicate that either (i) the amount of material ejected during nova eruptions in classical 
CVs is less than what is accreted and thus the WD mass increases during CV evolution or (ii) most CVs have undergone an early SSS phase during which a mass increase occurred.

In case (i), if the WDs can accrete enough mass to trigger an explosion, these could become SNe Ia with long delay times. In case (ii), the WDs would have already reached their maximum mass at the end of their SSS phase. These CVs could thus be seen as "failed SNe Ia". This view seems to be supported by far-UV observations of CVs showing that about $10 \%$ of systems exhibit a large N V / C IV emission line flux ratio, probably due to accretion of CNO-processed material (de Martino \& Gänsicke 2009).

\section{The Double Degenerate Channel}

According to the DD channel, two WDs merge and reach Chandrasekhar's limiting mass. The less massive of the two WDs is tidally disrupted and forms an accretion disk around its more massive companion. Carbon is then expected to ignite at the coreenvelope boundary of the accreting WD with the nuclear flame propagating inwards (e.g. Nomoto \& Iben 1985). This would turn the CO WD into an ONeMg WD. If the mass of the $\mathrm{ONeMg}$ WD reaches the Chandrasekhar limit, electron capture on $\mathrm{Ne}$ and $\mathrm{Mg}$ will cause an AIC of the WD into a neutron star. This seems to be the nearly inevitable fate of the merger of two WDs and has been the greatest stumbling block for this channel to be considered as a viable route to $\mathrm{SNe}$ Ia.

However, under very specific conditions, AIC may be avoided. If carbon ignites in the deep interior of the WD and is triggered in a way to switch from subsonic deflagration to supersonic detonation at the correct time, then one would obtain the observed explosion characteristics and the production of the correct mixture of elements.

Zhu, Chang \& van Kerkwijk (this volume) have explored how much fine tuning is necessary to produce those conditions that lead to SNe Ia for both Chandrasekhar and subChandrasekhar mass CO-CO WD mergers, and thus describe how central temperature, density, and merger remnant morphology critically depend on mass of the component stars and the mass ratio. They find that if mergers occur between roughly equal-mass CO WDs, the merger remnants are fully mixed, hottest at the center and surrounded by dense and small accretion discs. If the disk is accreted quickly, the compressional heating would likely lead to central carbon ignition at densities at which a detonation would lead to an SN Ia. With this merger scenario, the SNe Ia rates would be matched, since lower mass WDs could participate in the production of SNe Ia and would explain the observed range of luminosities and the fact that the bright SNe Ia are found in younger stellar populations (see also Sim et al., this volume).

Interestingly, Di Stefano has pointed out that most systems that evolve into DDs must also undergo a long-lived ( $>10^{6}$ years) NBWD phase as SSSs and thus are likely to spend a fraction of their lives as symbiotic systems. Hence we may still have to deal with the possibility that NBWDs may not appear as SSSs most of the time (Di Stefano, this volume), in order to reconcile their paucity with the existence of their progenies.

\subsection{DD Merger on a Dynamical Timescale}

Webbink pointed out that among double WDs massive enough to be SNe Ia candidates, the mass transfer process is nearly always dynamically unstable. That instability tidally disrupts the donor (less massive) WD within a few orbits, i.e., within a few minutes. Effective accretion rates can reach $10^{5} \mathrm{M}_{\odot}$ per year, far in excess of the nominal Eddington limit, so fast that photons have no time to diffuse, but are advected along with the gas flow. Much of the initial orbital angular momentum of the binary is carried off in tidal tails shed during disruption of the donor, and its disrupted remnant forms a 
rapidly rotating (but predominantly pressure-supported) envelope around the accreting WD. Shock heating may lead to carbon ignition at the base of this envelope, and, provided that burning is not quenched by expansion, it will percolate inward on a roughly thermal time scale. However, this process may be completely preempted by adiabatic compression of the accretor core, leading to immediate central CO ignition, triggering an SN Ia event. The calculations of Scannapieco, Raskin \& Timmes (this volume) based on adaptive mesh refinement simulations of DD mergers and collisions seem to (broadly speaking) support this scenario.

Crucial questions that need to be addressed are (i) the degree of internal heating by tidal dissipation in both accreting and donor WDs preceding the merger; (ii) the extent to which degeneracy of the donor may be lifted by accretion shocks during the merger process; (iii) what becomes of the remaining angular momentum of the merged WDs; and (iv) under what circumstances does compression of the accretor succeed or fail to lead to central carbon ignition. Detailed calculations are clearly needed to answer these questions.

\subsection{Surveys of Double WDs}

At this symposium, Marsh reviewed the observational evidence for populations of both detached and semi-detached DDs. Double WDs whose total mass is larger than the Chandrasekhar limit and that will merge through gravitational radiation within a Hubble time have long been proposed as possible SNe Ia progenitors. However, even though many surveys have been dedicated to their discoveries, (e.g. Bragaglia et al. 1990, Marsh, Dhillon \& Duck 1995, Napiwotzki, Christlieb, \& Drechsel 2003) no objects have so far been unequivocally identified. On the other hand, considering that only one double WD in one thousand needs to possess these characteristics to match the observed SNe Ia rates (Nelemans et al. 2001) and since only $\approx 1000$ WDs have so far been surveyed for close companions, the current lack of secure candidates is not as yet very significant.

Kilic et al. (2011) have undertaken an MMT survey of all previously identified extremely low-mass $(\mathrm{ELM})$ WDs $\left(M \sim 0.2 \mathrm{M}_{\odot}\right)$ from the SDSS DR4 to study their binary fraction and find possible mergers. They found 12 systems with a combined mass of $0.4<M / M_{\odot}<1.4$ that will merge within a Hubble time. The highest mass system is $\mathrm{J} 1233+1602$ with a combined mass of $1.37 \mathrm{M}_{\odot}$. These systems could evolve into a variety of objects such as under-luminous SNe Ia, extreme helium stars ( $\mathrm{RCrB})$, or single helium-enriched sub-dwarfs. They also remark that sub-Chandrasekhar explosions may not lead to "classical" SNe Ia but the number found seems to be consistent, according to Brown et al. (2011), with the observed rate of under-luminous SNe Ia. However, the calculations of Sim et al. (this volume) show that sub-Chandrasekhar explosions may in fact represent an important route to SNe Ia events (see $\S 3.3$ ).

\subsection{Sub-Chandrasekhar's Explosions}

SNe Ia could arise from detonations of sub-Chandrasekhar WDs, as reported by Sim et al. (this volume). They presented a numerical model that is relevant to any class of subChandrasekhar mass explosion, regardless of what triggers the detonation mechanism.

Sim et al. considered pure detonations of sub-Chandrasekhar CO WDs with different masses. For $0.97<M / M_{\odot}<1.15$ they obtained a ${ }^{56} \mathrm{Ni}$ yield of $\sim 0.3-0.8 M_{\odot}$ and were able to reproduce almost the full range of brightness observed in SNe Ia with rise and decline timescales and peak colors which are in good qualitative agreement with the observations of SNe Ia. The theoretical spectra corresponding to maximum light exhibit features due to intermediate-mass elements and match the correlation between the explosion luminosity and the ratio of the Si II lines at $\lambda 6355$ and $\lambda 5972$. 
Sub-Chandrasekhar models have the benefit that, because of the lower densities of these WDs, a detonation does not burn the entire WD into iron-peak elements. That is, these models do not need a finely tuned switch between deflagration and detonation. On the other hand, if the triggering mechanism is due to the ignition and detonation of a layer of helium, which would drive a shock propagating into the WD interior, then we are left with the following problem. $\mathrm{A} \sim 0.2 \mathrm{M}_{\odot}$ layer of helium would burn to ${ }^{56} \mathrm{Ni}$, giving early-time spectra rich in ${ }^{56} \mathrm{Ni}$, which are not observed. However, Bildsten et al. (2007) have shown that a detonation can occur in a helium layer of just $0.06-0.02 \mathrm{M}_{\odot}$ on $1.0-1.36 \mathrm{M}_{\odot} \mathrm{CO}$ WDs. Although their calculations did not follow the shock into the WD core, they speculated that it may drive a CO detonation in or near the stellar center. Guillochon et al. (2010) have also recently presented a new mechanism for the detonation of a sub-Chandrasekhar CO WD in a dynamically unstable system.

Sim et al. (this volume) point out that these could in fact be an important channel to SNe Ia, since the mergers of low-mass WDs would also come into play. These would yield much higher rates than those derived from Chandrasekhar mergers, as required by observations of SNe Ia birthrates.

\section{Core-Degenerate (CD) Channel}

Soker (this volume) proposed a new mechanism for the production of SNe Ia which consists of a merger, occurring at the end of the CE phase, between a WD and the degenerate core of its massive asymptotic giant branch companion star $\left(5-8 \mathrm{M}_{\odot}\right)$. This merger would occur while the core is still hot and relatively large and would form a centrifugally supported rapidly rotating WD with a mass that could exceed the Chandrasekhar mass limit. In this model, the delay from stellar formation to explosion would be determined by the spin-down time of the rapidly rotating merger remnant. Gravitational radiation was shown to be inefficient in spinning down the WD, while spin-down time-scales for magnetic-dipole radiation was shown to be consistent with the delay times required to explain SNe Ia.

Population synthesis calculations are needed to investigate whether the number of such systems is consistent with the birthrate of SNe Ia. Also, it is not completely clear whether the merged remnant, as it spins down and collapses, would instead yield a long $\gamma$-ray burst and a rapidly spinning magnetar (e.g. Tout et al. 2011). Long $\gamma$-ray bursts are usually associated with type $\mathrm{Ib} / \mathrm{c}$ SNe.

\section{Delay Time Distribution and Population Studies}

The delay time distribution (DTD) of SNe Ia is the rate at which SNe Ia occur after a stellar formation burst. Clearly, different progenitor scenarios yield different delay times between the birth of the binary system and the SN Ia event. In the SD channel, the time elapsed between the formation of the binary and the SN Ia explosion is given by the donor mass and thus by its main sequence lifetime. Under the DD scenario, in a younger universe the only WDs that could merge were the most massive ones originating from more massive progenitors. Consequently, a study of DTDs provides an excellent method to investigate all possible progenitor channels.

Recent observations of SNe Ia indicate that their physical parameters depend on the type of host galaxy. SNe Ia are more common (by at least one order of magnitude per unit stellar mass), intrinsically more luminous, and with wider light-curves in late-type galaxies with ongoing star-formation than they are in elliptical galaxies (e.g. Mannucci 
et al. 2005). Sub-luminous SNe Ia are found mainly in galaxies with older stellar populations. They also appear to occur exclusively in massive galaxies. This is in stark contrast to "normal" SNe Ia which occur across more diverse types of hosts. As a consequence, it is to be expected that brighter and wider light-curve SNe Ia should be more common in the distant universe than they are in the local universe (Howell et al. 2007). Thus, all the available observational evidence seems to indicate that the local SN Ia samples are different from the high redshift ones. These redshift-related discrepancies are of concern to cosmological studies, since these depend on SNe Ia being reliable "standard candles". It is therefore important to establish whether the same light curve "stretching" that is calibrated and used at low redshfits is also applicable at high redshifts (see Greggio 2010).

Scannapieco \& Bildsten (2005), Mannucci, Della Valle, \& Panagia (2006), and Sullivan et al. (2006) suggested that these effects may point to the coexistence of more than one class of SNe Ia progenitors. In particular, they showed that the delay-time distributions with both "prompt" (100-500 Myr) and "delayed" ( 5 Gyr) components, or with a wide range of delay-times, fit the observations quite well. Pritchet reported at this symposium a DTD with a steeper power-law slope, and found differences in the DTD as a function of light curve characteristics which may also point to the coexistence of more than one class of SNe Ia progenitors.

In this context, the studies of Townsley et al. (2009) are very interesting, since they show how the metallicity and the average age of the parent stellar population can influence the characteristics of SNe Ia. In particular, they find (in agreement with observations) that the average luminosity of SNe Ia should decrease with stellar population age, since colder WDs have higher core densities and produce less ${ }^{56} \mathrm{Ni}$ (possibly due to a higher rate of neutronization).

In summary, the importance of the analysis of the DTDs at higher redshifts cannot be emphasized enough, since it will help us understand how the DTD evolves over time. To this purpose, Regös et al. (this volume) are planning a survey to investigate SNe Ia at redshifts up to $z \sim 2$.

\section{Conclusions}

Almost $80 \%$ of SNe Ia are "normal" in the sense that they are virtually carbon copies of each other, while the remaining $20 \%$ appear quite different and can be described as "extremely weak", "weak" or "extremely luminous". The spread in observed peak luminosities, differences in light curve characteristics, delay time distributions, and the dependence of physical parameters on the type of host galaxy all appear to suggest that more than one channel may be responsible for SNe Ia.

Discussions of the "normal" SNe Ia were focused on the explosion properties of Chandrasekhar mass WDs where a WD is driven to the Chandrasekhar limit by accretion in a binary system. There was no consensus on the nature of the binary star progenitor, although most participants appeared to favor the SD hypothesis. The SD hypothesis requires the system to go through a phase of stable nuclear burning on the surface of the WD, but the known number of SSSs leads to estimates for birth rates of SNe Ia that fall short of the required birth rate by several orders of magnitude. Either the majority of NBWDs radiate at other wavelengths, or SD progenitors only contribute a small fraction of the total SNe Ia rate. This, together with the lack of evidence of hydrogen in the spectra of these systems were discussed as major problems for the model from an observational point of view. A criticism of the SD model was that it had a "lot of moving parts" and could only explain the observed delay time $\propto t^{-1}$ in a "contrived way", while 
this relationship was explained more naturally in the DD models (see the calculations of Ruiter, Belczynski \& Fryer 2009).

As has been known for sometime, all Chandrasekhar explosion models require an ad hoc transition between the time of deflagration and detonation to generate light and abundance curves that agree with observations. None of the papers presented at the conference yielded new theoretical insights on this problem. For the DD channel to work there was the additional constraint that the accreting CO WD should detonate at the center, otherwise it would be transformed into an ONeMg WD which would then collapse into a neutron star via an AIC. Again, there were no calculations presented at the conference that directly addressed this problem although there was some discussion led by Webbink around the idea that in the DD channel compressional heating of the core caused by very high accretion rates from a dynamically unstable accretion disk may play a dominant role in triggering central detonation.

A highlight of the conference was perhaps the demonstration that detonation models could be constructed for WDs with masses ranging from sub-Chandrasekhar to Chandrasekhar. These could explain the entire range of SNe Ia light curve and spectral characteristics from extremely weak through normal SNe Ia, opening up a variety of possibilities for DD models. However, this model also suffers from the lack of an easily identifiable triggering mechanism.

All in all, it is fair to say that despite the significant progress made on both observational and theoretical fronts, the nature of the progenitors of SNe Ia still remains unresolved.

\section{References}

Belczynski, K. \& Mikolajewska, J. 1998, MNRAS, 296, 77

Bildsten, L., Shen, K. J.,Weinberg, N. N., \& Nelemans, G. 2007, ApJ, 662, L95

Bragaglia, A., Greggio, L., Renzini, A., \& D'Odorico, S. 1990, ApJ, 365, L13

Brown, W. R., Kilic, M., Allende Prieto, C., \& Kenyon, S. J. 2011, MNRAS, 411, L31

de Martino, D. \& Gnsicke, B. T. 2009, Ap\&SSS, 320, 135

Di Stefano, R., Voss, R., \& Claeys, J. S. W. 2011, ApJ, 738, L1

Greggio, L. 2010, MNRAS, 406, 22

Guillochon, J., Dan, M., Ramirez-Ruiz, E., \& Rosswog, S. 2010, ApJ, 709, L64

González Hernández, J. I., Ruiz-Lapuente, P., Filippenko, A. V., Foley, R. J., Gal-Yam, A., \& Simon, J. D. 2009, ApJ, 691, 1

Howell, D., et al. 2006, Nature, 443, 308

Howell D. A., Sullivan M., Conley A., \& Carlberg R. 2007, ApJ, 667, L37

Hurley, J. R., Tout, C. A., Wickramasinghe, D. T., Ferrario, L., \& Kiel, P. D. 2010, MNRAS, 402, 1437

Iben, I., Nomoto, K., Tornambe, A., \& Tutukov, A. V. 1987, ApJ, 317, 717

Iben, I. \& Tutukov, A. V. 1987, ApJS, 54, 335

Justham, S., Wolf, C., Podsiadlowski, Ph., \& Han, Zh. 2009, A\&SA, 493, 1081

Kilic, M., Brown, W. R., Allende Prieto, C., Agüeros, M. A., Heinke, C., \& Kenyon, S. J. 2011, ApJ, 727, 3

Mannucci, F., Della Valle, M., Panagia, N., Cappellaro, E., Cresci, G., Maiolino, R., Petrosian, A., \& Turatto, M. 2005, A\&A A, 433, 807

Mannucci F., Della Valle M., \& Panagia N. 2006, MNRAS, 370, 773

Marietta, E., Burrows, A., \& Fryxell, B. 2000, ApJS, 128, 615

Marsh, T. R., Dhillon, V. S., \& Duck, S. R. 1995, MNRAS, 275, 828

Napiwotzki, R., Christlieb, N., Drechsel, H., et al. 2003, ESO Msngr, 112, 25

Nelemans, G., Yungelson, L. R., Portegies Zwart, S. F., \& Verbunt, F. 2001, A\&\&A, 365, 491

Nelson, T., Mukai, K., Orio, M., Luna, G. J. M., \& Sokoloski, J. L. 2011, ApJ, in press 
Nomoto, K. \& Iben, I. 1985, ApJ, 297, 531

Podsiadlowski, Ph. 2003, astro-ph/0303660v1

Ruiter, A. J., Belczynski, K., \& Fryer, C. 2009, ApJ, 699, 2026

Ruiz-Lapuente, P., et al. 2004, Nature, 431, 1069

Scannapieco E. \& Bildsten L. 2005, ApJ, 629, L85

Sullivan M., et al. 2006, ApJ, 648, 868

Totani, T., Morokuma, T., Oda, T., Doi, M., \& Yasuda, N. 2008, PASJ, 60, 132

Tout, C. A., Wickramasinghe, D. T., Lau, Herbert H.-B., Pringle, J. E., \& Ferrario, L. 2011, MNRAS, 410, 2458

Townsley, D. M., Jackson, A. P., Calder, A. C., Chamulak, D. A., Brown, E. F., \& Timmes, F. X. 2009, ApJ, 701, 1582

Trümper J., Hasinger, G., Aschenbach, B., Bräuninger, H., Briel, U. G., Burkert, W., Fink, H., Pfeffermann, E., Pietsch, W., Predehl, P., Schmitt, J. H. M. M., Voges, W., Zimmermann, U., \& Beuermann, K. 1991, Nature, 349, 579

Webbink, R. F. 1984, ApJ, 277, 355

Whelan, J. \& Iben, I. 1973, ApJ, 186, 1007

Woosley, S. E. \& Weaver, T. A. 1994, ApJ, 423, 371

Yoon, S.-C., Podsiadlowski, P., \& Rosswog, S. 2007, MNRAS, 380, 933

Zorotovic, M., Schreiber, M. R., \& Gänsicke, B. T. 2011, A\&̊A, 536, 42 